\title{
Video-assisted thoracoscopic surgery using mobile computed tomography: New method for locating of small lung nodules
}

\author{
Kazuto Ohtaka ${ }^{1 *}$, Yasuhiro Takahashi ${ }^{1}$, Kichizo Kaga ${ }^{2}$, Naoto Senmaru ${ }^{1}$, Yoshihisa Kotani ${ }^{3}$ and Yoshiro Matsui $^{2}$
}

\begin{abstract}
Background: The O-arm is an intraoperative imaging device that can provide computed tomography images. Surgery for small lung tumors was performed based on intraoperative computed tomography images obtained using the O-arm. This study evaluated the usefulness of the O-arm in thoracic surgery.

Methods: From July 2013 to November 2013, 10 patients with small lung nodules or ground glass nodules underwent video-assisted thoracoscopic surgery using the O-arm. A needle was placed on the visceral pleura near the nodules. After the lung was re-expanded, intraoperative computed tomography was performed using the O-arm. Then, the positional relationship between the needle marking and the tumor was recognized based on the intraoperative computed tomography images, and lung resection was performed.

Results: In 9 patients, the tumor could be seen on intraoperative computed tomography images using the O-arm. In 1 patient with a ground glass nodule, the lesion could not be seen, but its location could be inferred by comparison between preoperative and intraoperative computed tomography images. In only 1 patient with a ground glass nodule, a pathological complete resection was not performed. There were no complications related to the use of the O-arm.
\end{abstract}

Conclusions: The O-arm may be an additional tool to facilitate intraoperative localization and surgical resection of non-palpable lung lesions.

Keywords: O-arm, Lung, Video-assisted thoracoscopic surgery, Ground glass nodules

\section{Background}

To perform surgery for small lung tumors that include ground glass nodules (GGNs), preoperative or intraoperative procedures to identify the locations of the tumors are generally needed. The preoperative marking procedures principally consist of two methods: the percutaneous method and the transbronchial method. The percutaneous method involves computed tomography (CT)-guided placement of various marking materials, including hook-wire, vital dye, and radioactive material [1-5]. The transbronchial method involves bronchoscopyguided placement of various marking materials, including metallic coils and barium [6]. These preoperative marking methods require intraoperative fluoroscopic guidance to

\footnotetext{
*Correspondence: kit_katton0803@yahoo.co.jp

'Department of Thoracic Surgery, Steel Memorial Muroran Hospital,

Chiribetsu-cho, 050-0076 Muroran, Hokkaido, Japan

Full list of author information is available at the end of the article
}

localize the marking materials. However, these methods have some disadvantages. The percutaneous method has a limitation with respect to the puncture site, as well as some serious complications (pneumothorax, dissemination, and air embolism) [7]. The transbronchial method sometimes needs advanced bronchoscopy techniques. Additionally, because the lung is collapsed during surgery, intraoperative positional relationships between a marking material and the lesion may differ from the preoperative positional relationships, and it may cause incomplete resection. Other methods to localize the tumors have been reported, such as intraoperative ultrasonography and the intrathoracic stamping method [8-10].

The O-arm Surgical Imaging System (Medtronic Japan Co., Ltd., Tokyo, Japan) is an intraoperative, full-rotation, multidimensional image system that functions as an intraoperative imaging device with a flat-panel detector that provides two-dimensional (2D) fluoroscopic imaging and 
three-dimensional (3D) cone-beam CT imaging (Figure 1). This system has been used in spine, orthopedic, and trauma-related surgeries, and has recently also been used in neurosurgery [11-13]. In these surgeries, after the navigation imaging is constructed based on intraoperative CT images using the $\mathrm{O}$-arm, the surgery is performed using these navigation images. Petrov et al. suggested that the $\mathrm{O}$-arm could be used for not only bone tissue but also for soft tissue [14]. Therefore, we hypothesized that the O-arm could be used for small lung tumors.

In this study, surgery for small lung tumors that included GGNs was performed based on intraoperative $\mathrm{CT}$ images using the $\mathrm{O}$-arm. This is the first report using the $\mathrm{O}$-arm in thoracic surgery.

\section{Methods}

The Steel Memorial Muroran Hospital Institutional Review Board approved this study. Informed consent was obtained from the patients for the publication of this report and any accompanying images.

\section{Patients}

From July 2013 to November 2013, 23 patients with a small lung tumor underwent video-assisted thoracoscopic surgery (VATS) in Steel Memorial Muroran Hospital. Of these 23 patients, 10 patients with small lung lesions underwent the surgery using the $\mathrm{O}$-arm.

\section{O-arm}

The O-arm Surgical Imaging System was used. This system can provide 2D fluoroscopic imaging and 3D conebeam CT imaging. Cone-beam CT images are acquired in standard mode (standard definition), where about 400 projection views over 360 degrees are acquired in 13 seconds. The 3D imaging volumes, which have a diameter of $20 \mathrm{~cm}$ and a length of $15 \mathrm{~cm}$, are reconstructed with $512 \times 512 \times 192$ voxels $(0.830 \mathrm{~mm}$ axial, coronal, and sagittal slice thicknesses) in approximately 20 seconds. The dose length projection ranges 140 to 320 mGycm depending on patient size.

\section{Indication for localization using the $\mathrm{O}$-arm}

In our institute, clinical indication for preoperative localization were on the basis of the following preoperative CT findings: (1) maximum diameter of nodule of $10 \mathrm{~mm}$ or less, (2) minimum distance between the visceral pleura and superior border of the nodule of $10 \mathrm{~mm}$ or more, (3) GGN which had no contact with visceral pleura. So we considered that the $\mathrm{O}$-arm would be eventually used for these lesions. However, because there had been no experience using the $\mathrm{O}$-arm for lung tumors in thoracic surgery, the $\mathrm{O}$-arm was used for small tumors that were predicted to be in easily recognized locations in this study.

\section{Surgical Technique}

Sublober resection was performed by VATS with a 3-cm access thoracotomy incision and 2 ports. When both lobectomy and sublober resection in a different lobe was performed, it was performed by VATS with a $7-\mathrm{cm}$ thoracotomy incision, a 3-cm small thoracotomy incision, and 2 ports.

Under general anesthesia with a double-lumen tube in the lateral position, povidone-iodine was applied to the patient who was wrapped in a sterile drape. The O-arm system was wrapped in a plastic drape and bought into

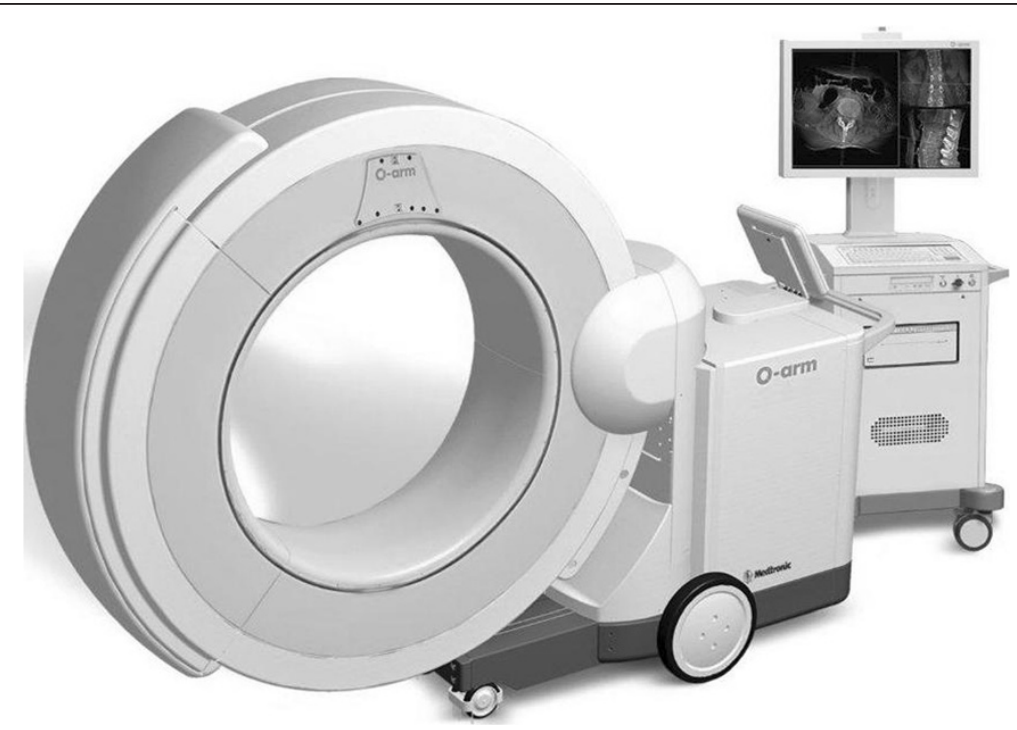

Figure 10 -arm surgical imaging system. 
the operation field. The operation table used was a normal type, not a special type.

First, to decrease frequency of the O-arm scan, the needle marking was put on the visceral pleura lying directly on the lesion using the intrathoracic stamping method reported by Kawada et al. [10]. A mark was put on the skin at the shortest distance from the lesion based on preoperative CT images scanned at maximal inspiration. An injection needle was inserted vertically from the skin mark through the chest wall into the pleural cavity. Nylon thread was inserted through an injection needle into the pleural cavity and withdrawn from the pleural cavity through a small thoracotomy. A small gauze ball containing indigo carmine dye (Daiichi Sankyo Co., Ltd., Tokyo, Japan) was tied to the nylon thread and pulled back into the pleural cavity. The gauze ball was tugged towards the internal surface of the thoracic wall, where it was anchored. After the lung was re-expanded, the dye from the gauze ball stamped the visceral pleura. After the lung was then collapsed again, a needle with 4-0 polydiaxonone (PDS) thread was placed near the stamp on the visceral pleura.

Second, the O-arm scan was performed. The O-arm was positioned to the target level of the needle marking based on the 2D fluoroscopic images using the O-arm. After the lung was re-expanded, the O-arm 3D scan was performed at maximal inspiration, and $\mathrm{CT}$ images were reconstructed.

Third, the positional relationship between the lesion and the needle marking was determined based on these $\mathrm{CT}$ images. If the needle marking was away from the lesion, the needle with 4-0 PDS thread was again placed near the lesion based on the first intraoperative CT images. The O-arm 3D scan was then performed again.

Finally, lung resection was performed based on the intraoperative CT images.

The time for the set-up of the O-arm was about $5 \mathrm{mi}$ nutes. The time for intraoperative use of the $\mathrm{O}$-arm was about 10 to 15 minutes.

All medical staff in the operation room wore radioprotectors. When the $\mathrm{O}$-arm 3D scan was performed, the anesthesiologist went behind the radioprotective lead screen near the anesthesia equipment, and the radiological technologist and the surgeon went behind the operator's console, where was expected to have exposure of less than $0.1 \mathrm{mR}$ per spin. Other paramedical staff left the operating room during the $\mathrm{O}$-arm $3 \mathrm{D}$ scan and returned immediately after the $3 \mathrm{D}$ scan.

\section{Results}

The characteristic of the 10 patients are shown in Table 1 . The pathological diagnoses were 4 primary cancers, 5 metastatic tumors, and 1 benign tumor. The preoperative CT findings were 5 solid lesions and 5 GGNs.
Table 1 Patients' characteristics

\begin{tabular}{|c|c|}
\hline & $\mathrm{N}=10$ \\
\hline Age, median value (range), years & $66(52-80)$ \\
\hline \multicolumn{2}{|l|}{ Sex, $n$} \\
\hline Male/Female & $5 / 5$ \\
\hline \multicolumn{2}{|l|}{$\mathrm{CT}$ findings, $\mathrm{n}$} \\
\hline Solid lesions/Ground grass nodules & $5 / 5$ \\
\hline \multicolumn{2}{|l|}{ Lesion of tumor, $\mathrm{n}$} \\
\hline Right upper lobe/Right lower lobe & $3 / 3$ \\
\hline Left upper lobe/Left lower lobe & $3 / 1$ \\
\hline \multicolumn{2}{|l|}{ Diagnosis } \\
\hline Primary cancer/Metastatic tumor/benign tumor & $4 / 5 / 1$ \\
\hline Size of tumor, median value (range), $\mathrm{mm}$ & $10(6-14)$ \\
\hline \multicolumn{2}{|l|}{ Operative procedure, $\mathrm{n}$} \\
\hline Sublober resection/Lobectomy with sublober resection & $8 / 2$ \\
\hline Operative time, median value (range), minutes & $106(58-243)$ \\
\hline Blood loss, median value (range), $\mathrm{mL}$ & $3(0-100)$ \\
\hline \multicolumn{2}{|l|}{ Postoperative complication, $\mathrm{n}$} \\
\hline Pulmonary fistula & 3 \\
\hline
\end{tabular}

The median size of the resected tumor was $10 \mathrm{~mm}$ (range, 6-14 mm). The operative procedures were $8 \mathrm{sub}$ lober resections and 2 both lobectomy and sublober resection in a different lobe. Of the 8 patients with sublober resection, 1 patient underwent resection for 6 lesions, 1 patient underwent resection for 2 lesions, and the remaining 6 patients underwent resection for 1 lesion. The median operative time for all 10 patients was 105 minutes (range, 58-243 minutes). The median operative time for the 6 patients with sublober resection of a lesion was 67 minutes (range, 58-110 minutes).

In 9 patients, the tumor could be seen on intraoperative CT images using the $\mathrm{O}$-arm (Figure 2). Of them, in 1 patient with a GGN, because the lung was incompletely re-expanded, intraoperative $\mathrm{CT}$ using the $\mathrm{O}$-arm showed a higher density lesion than the preoperative CT, similar to a solid tumor (Figure 3).

In 1 patient with a GGN, the tumor could not be seen, but its location could be inferred by comparing the positions of the peripheral pulmonary vessels or the bronchus near the tumor on the preoperative and intraoperative $\mathrm{CT}$ images (Figure 4). Additionally, a small nodule, even $3 \mathrm{~mm}$ in diameter, was accidentally scanned and could be seen obviously by the $\mathrm{O}$-arm (Figure 5).

The O-arm scan was performed 1 time in 4 patients, 2 times in 4 patients, 3 times in the 1 patient in whom the tumor could not be seen on intraoperative CT, and 5 times in the 1 patient who underwent resections for 6 lesions.

In 9 patients, pathological complete resection was achieved; in only 1 patient with a GGN, a pathological 


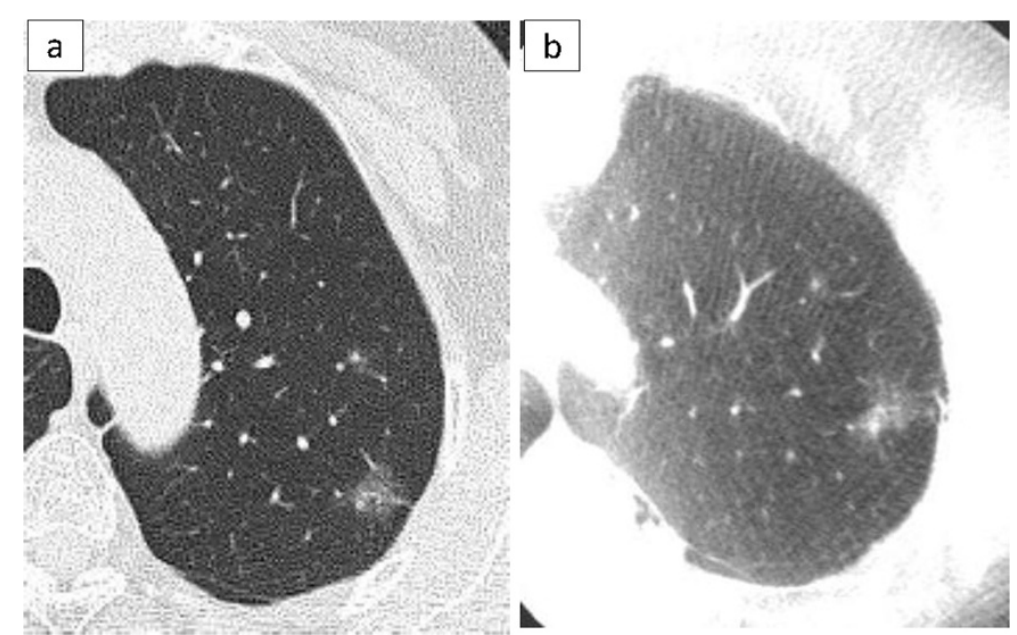

Figure 2 A GGN with a solid part. Preoperative CT shows a GGN, $14 \mathrm{~mm}$ in diameter, with a small solid part in the left upper lobe (a). The O-arm shows the same finding as the preoperative $C T$ (b).

complete resection was not achieved. This might have been caused by a technical error during resection. This patient underwent additional resection later.

There were no complications related to the use of the O-arm.

\section{Discussion}

Surgery for small lung tumors that included GGNs was performed based on intraoperative CT images using the $\mathrm{O}$-arm. In our experience, a small nodule, $3 \mathrm{~mm}$ in diameter, could be easily seen on intraoperative CT images using the $\mathrm{O}$-arm. Thus, the $\mathrm{O}$-arm may be able to show even very small solid tumors. A GGN could be obviously seen on intraoperative $\mathrm{CT}$ images using the $\mathrm{O}$-arm. A
GGN without a solid part could not be seen in 1 patient. However, the location of the tumor could be inferred by comparing the positions of the peripheral pulmonary vessels or the bronchus near the tumor on the preoperative and intraoperative $\mathrm{CT}$ images. Because the $\mathrm{O}$-arm can provide much the same $\mathrm{CT}$ images as the preoperative $\mathrm{CT}$ images, the location of the tumor can be inferred by comparison between preoperative and intraoperative CT images if the tumor is not shown on intraoperative CT images using the $\mathrm{O}$-arm. Additionally, using the $\mathrm{O}$-arm when the lung was incompletely re-expanded, a GGN could be shown as a higher density lesion than on the preoperative CT images. The O-arm may make it possible to perform surgery for small, non-palpable lung lesions.

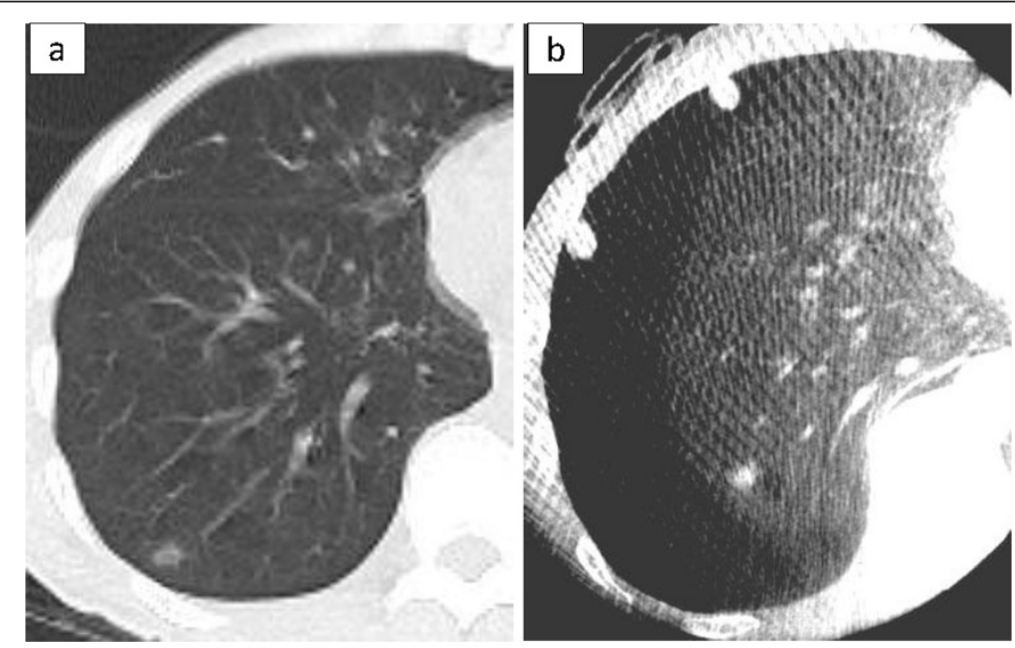

Figure 3 A GGN without a solid part. Preoperative $C T$ shows a GGN, 9 mm in diameter, without a solid part (a). When the lung is incompletely re-expanded, the O-arm shows a higher density lesion than the preoperative CT finding, similar to a solid tumor (b). 

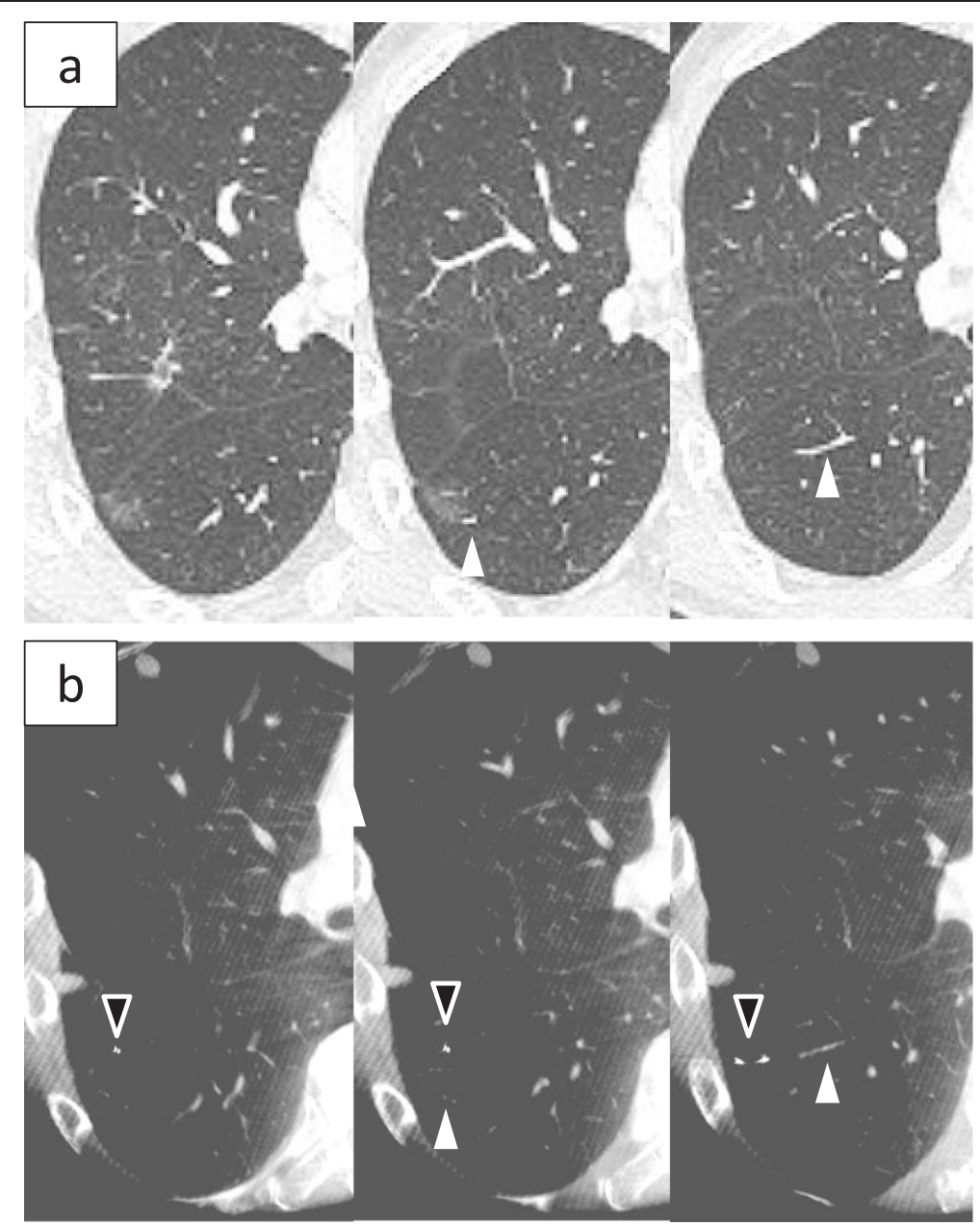

Figure 4 A GGN without a solid part. Preoperative CT shows a GGO lesion, $10 \mathrm{~mm}$ in diameter, without a solid part (a). The O-arm could not show the lesion. However, the location of the tumor could be inferred by comparing the positions of the peripheral pulmonary vessels (white arrowhead) or the bronchus near the tumor on the preoperative and intraoperative CT images (b). A PDS needle is placed as a marker (black arrowhead).

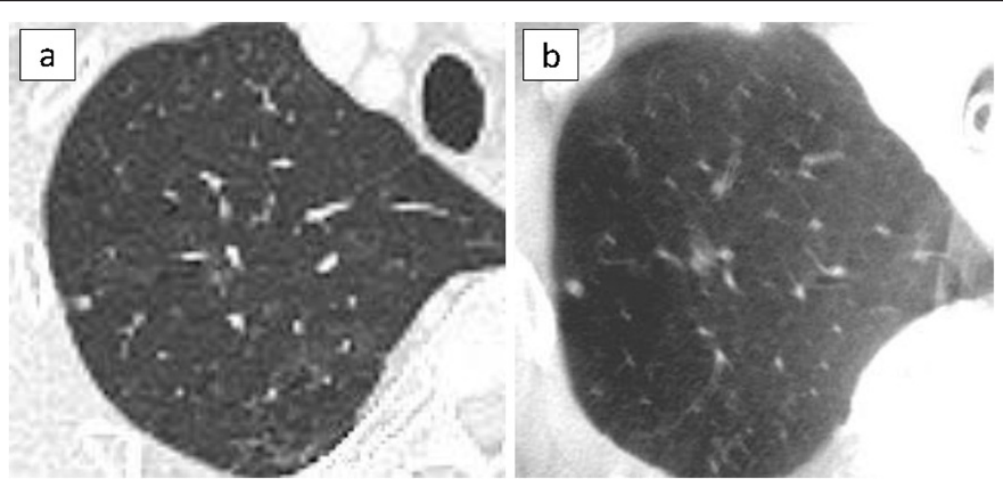

Figure 5 A small nodule. Preoperative CT shows a small nodule, $3 \mathrm{~mm}$ in diameter (a). The O-arm shows an obvious nodule (b). 
In our experiences, only 1 patient with a GGN could not be seen the tumor on the intraoperaive CT images. This might have been due to technical difficulties with scan conditions. Based on our experiences using the $\mathrm{O}$ arm, if the $\mathrm{O}$-arm was positioned under the condition that the tumor was located in the center of the $\mathrm{O}$ shape arm, the tumor could be obviously seen on the intraoperative CT images. However, if the $\mathrm{O}$-arm was positioned under the condition that the tumor was not located in the center of the $\mathrm{O}$ shape arm, the tumor might not be seen. After we experienced the patient who the tumor could not be seen on the intraoperative CT images, we adjusted the $\mathrm{O}$-arm position under the condition that the tumor was located in the center of the $\mathrm{O}$ shape arm and did not experience that case.

There are several benefits in surgery using the O-arm. First, it is not necessary to perform any type of preoperative marking procedure. Preoperative marking procedures, such as the percutaneous method and the transbronchial method, can be difficult for patients and can cause serious complications as pneumothorax, dissemination, and air embolism that may result in the delay or cancellation of surgery [7]. Second, there is a limit to the tumor locations that can be marked with preoperative marking procedures, but there is no such limit with the $\mathrm{O}$-arm.

The disadvantage in surgery using the O-arm is the exposure to radiation. Medtronic Inc. has reported that the effective whole body dose using the $\mathrm{O}$-arm standard $3 \mathrm{D}$ protocols for the chest was lower than for 8, 16, and 64-slice CT [15]. In a report dealing with orthopedic surgery, the mean radiation dose of the O-arm 3D scan was comparable to that of half the dose of a 64 multislice CT scan [16]. We estimated that the O-arm resulted in lower radiation exposure than conventional CT in thoracic surgery. However, when it is compared to preoperative marking method, it remains unclear which has higher total radiation exposure for patients.

In our experience, a needle marking was put on the visceral pleura around the tumor based on preoperative skin marking, which was placed based on preoperative CT images. If the preoperative skin marking was not near the tumor, the first needle marking on the visceral pleura was also not near the tumor, and intraoperative $\mathrm{CT}$ scanning using the $\mathrm{O}$-arm was needed more than once. To decrease the frequency of intraoperative CT scans and radiation exposure, it is necessary to place the skin marking with more precision.

To reduce radiation exposure for medical staff, when the $\mathrm{O}$-arm scan was performed, medical staffs wearing a radioprotector went behind the radioprotective lead screen or got away from the $\mathrm{O}$-arm. In a report involving orthopedic surgery, the $\mathrm{O}$-arm resulted in less radiation exposure to the surgeon than the C-arm [17]. We considered that there was no major problem with radiation exposure for medical staff when appropriate measures were used with the $\mathrm{O}$-arm.

\section{Conclusions}

This is the first report of the use of the O-arm-which can be used to perform intraoperative $\mathrm{CT}$ and has been used in orthopedic surgery-in thoracic surgery. The O-arm may be an additional tool to facilitate intraoperative localization and surgical resection of non-palpable lung lesions.

\section{Abbreviations}

GGN: Ground glass nodule; VATS: Video-assisted thoracoscopic surgery.

\section{Competing interests}

The authors declare that they have no competing interests.

\section{Authors' contributions}

$\mathrm{KO}$ performed the study design, the acquisition of data, and the analysis, and drafted the manuscript. YT, KK, NS,YK and YM helped to draft the manuscript. All authors read and approved the final manuscript.

\section{Acknowledgement}

The authors thank Hirokatsu Kato and Yu Fang Qin (Department of Anesthesiology, Steel Memorial Muroran).

\section{Author details}

${ }^{1}$ Department of Thoracic Surgery, Steel Memorial Muroran Hospital, Chiribetsu-cho, 050-0076 Muroran, Hokkaido, Japan. ²Department of Cardiovascular and Thoracic Surgery, Hokkaido University Graduate School of Medicine, Sapporo, Hokkaido, Japan. ${ }^{3}$ Department of Orthopedic Surgery, Steel Memorial Muroran Hospital, Muroran, Hokkaido, Japan.

Received: 15 April 2014 Accepted: 12 June 2014

Published: 20 June 2014

\section{References}

1. Sugi K: Radioisotope marking under CT guidance and localization using a handheld gamma probe for small or indistinct pulmonary lesions. Chest 2003, 124:155-1558.

2. Lenglinger FX, Schwarz CD, Artmann W: Localization of pulmonary nodules before thoracoscopic surgery: Value of percutaneous staining with methylene blue. AJR 1994, 163:297-300.

3. Ciriaco P, Negri G, Puglisi A, Nicoletti R, Maschio AD, Zannini P: Videoassisted thoracoscopic surgery for pulmonary nodules: rationale for preoperative computed tomography-guided hookwire localization. Eur J Cardiothorac Surg 2004, 25:429-433.

4. Powell TI, Jangra D, Clifton JC, Lara-Guerra H, Church N, English J, Evans K, Yee J, Coxson H, Mayo JR, Finley RJ: Peripheral lung nodules. Fluoroscopically guided video-assisted thoracoscopic resection after computed tomographyguided localization using platinum microcoils. Ann Surg 2004, 240:481-489.

5. Watanabe K, Nomori H, Otsuka T, Kaji M, Naruke T, Suemasu K: Usefulness and complications of computed tomography-guided lipiodol marking for fluoroscopy-assisted thoracoscopic resection of small pulmonary nodules: experience with 174 nodules. J Thorac Cardiovasc Surg 2006, 132:320-324.

6. Asano F, Shindoh J, Shigemitsu K, Miya K, Abe T, Horiba M, Ishihara Y: Ultrathin bronchoscopic barium marking with virtual bronchoscopic navigation for fluoroscope-assisted thoracoscopic surgery. Chest 2004, 126:1687-1693.

7. Sakiyama S, Kondo K, Matsuoka H, Yoshida M, Miyoshi T, Yoshida S, Monden Y: Fatal air embolism during computed tomography-guided pulmonary marking with a hook-type marker. I Thorac Cardiovasc Surg 2003, 126:1207-1209.

8. Santambrogio R: Intraoperative ultrasound during thoracoscopic procedures for solitary pulmonary nodules. Ann Thorac Surg 1999, 68:218-222 
9. Sortini A, Carrella G, Sortini D, Pozza E: Single pulmonary nodules: localization with intrathoracoscopic ultrasound. A prospective study. Eur J Cardiothorac Surg 2002, 22:440-442.

10. Kawada M, Okubo T, Poudel S, Suzuki Y, Kawarada Y, Kitashiro S, Okushiba S, Katoh H: A new marking technique for peripheral lung nodules avoiding pleural puncture: the intrathoracic stamping method. Interact CardioVasc Thorac Surg 2013, 16:381-383.

11. Collins KL, Patil PG: Flat-panel fluoroscopy O-arm-guided percutaneous radiofrequency cordotomy: a new technique for the treatment of unilateral cancer pain. Neurosurgery 2013, 72:27-34.

12. Holloway K, Docef A: A quantitative assessment of the accuracy and reliability of O-arm images for deep brain stimulation surgery. Neurosurgery 2013, 72:47-57.

13. Costa F, Tomei M, Sassi M, Cardia A, Ortolina A, Servello D, Fornari M: Evaluation of the rate of decompression in anterior cervical corpectomy using an intra-operative computerized tomography scan (O-Arm system). Eur Spine J 2012, 21:359-363.

14. Petrov IE, Nikolov HN, Holdsworth DW, Drangova M: Image performance evaluation of a 3D surgical imaging platform. Proc SPIE 2011, 7961:796150.

15. Medtronic Navigation, Inc: O-ARM 1000 Dosimetry Report, Document Nr: B1-700-00027-041 Rev01. 2008.

16. Van de Kelft E, Costa F, Van der Planken D, Schils F: A prospective multicenter registry on the accuracy of pedicle screw placement in the thoracic, lumber, and sacral levels with the use of the O-arm Imaging System and StealthStation Navigation. Spine 2012, 37:E1580-E1587.

17. Tabaraee E, Gibson AG, Karahalios DG, Potts EA, Mobasser JP, Burch S: Intraoperative cone beam computed tomography with navigation (O-arm) versus conventional fluoroscopy (C-arm): A cadaveric study comparing accuracy, efficiency, and safety for spinal instrumentation. Spine 2013, 38:1953-1958.

doi:10.1186/1749-8090-9-110

Cite this article as: Ohtaka et al.: Video-assisted thoracoscopic surgery using mobile computed tomography: New method for locating of small lung nodules. Journal of Cardiothoracic Surgery 2014 9:110.

\section{Submit your next manuscript to BioMed Central and take full advantage of:}

- Convenient online submission

- Thorough peer review

- No space constraints or color figure charges

- Immediate publication on acceptance

- Inclusion in PubMed, CAS, Scopus and Google Scholar

- Research which is freely available for redistribution 